\title{
HINODE/EIS SPECTROSCOPIC VALIDATION OF VERY HOT PLASMA IMAGED WITH THE SOLAR DYNAMICS OBSERVATORY IN NON-FLARING ACTIVE REGION CORES
}

\author{
PaOla Testa ${ }^{1}$ and Fabio Reale ${ }^{2,3}$ \\ ${ }^{1}$ Smithsonian Astrophysical Observatory, 60 Garden street, MS 58, Cambridge, MA 02138, USA; ptesta@ cfa.harvard.edu \\ ${ }^{2}$ Dipartimento di Fisica, Università di Palermo, Piazza del Parlamento 1, 90134 Palermo, Italy \\ ${ }^{3}$ INAF-Osservatorio Astronomico di Palermo, Piazza del Parlamento 1, 90134 Palermo, Italy \\ Received 2012 February 27; accepted 2012 March 28; published 2012 April 11
}

\begin{abstract}
We use coronal imaging observations with the Solar Dynamics Observatory/Atmospheric Imaging Assembly (AIA), and Hinode/Extreme-ultraviolet Imaging Spectrometer (EIS) spectral data to explore the potential of narrowband EUV imaging data for diagnosing the presence of hot $(T \gtrsim 5 \mathrm{MK})$ coronal plasma in active regions. We analyze observations of two active regions (AR 11281, AR 11289) with simultaneous AIA imaging and EIS spectral data, including the Ca XVII line (at 192.8 $\AA$ ), which is one of the few lines in the EIS spectral bands sensitive to hot coronal plasma even outside flares. After careful co-alignment of the imaging and spectral data, we compare the morphology in a three-color image combining the 171, 335, and 94 A AIA spectral bands, with the image obtained for Ca XVII emission from the analysis of EIS spectra. We find that in the selected active regions the Ca XVII emission is strong only in very limited areas, showing striking similarities with the features bright in the $94 \AA$ (and $335 \AA$ ) AIA channels and weak in the $171 \AA$ A band. We conclude that AIA imaging observations of the solar corona can be used to track hot plasma (6-8 MK), and so to study its spatial variability and temporal evolution at high spatial and temporal resolution.
\end{abstract}

Key words: Sun: activity - Sun: corona - Sun: UV radiation - Sun: X-rays, gamma rays - techniques: imaging spectroscopy

Online-only material: color figure

\section{INTRODUCTION}

In spite of remarkable progress, and although it is well established that the ultimate energy source is the coronal magnetic field, the question of how magnetic energy is transformed to heat the coronal plasma is still to be solved (e.g., Klimchuk 2006; Reale 2010). Among the several aspects debated in the literature, one important issue is whether the heating is released gradually and continuously or in the form of discrete, rapid, and intense pulses. Several physical and technical reasons inhibit a direct answer, e.g., the efficient thermal conduction along the magnetic field lines, the low emission from the heating site, and limited spatial and temporal resolution (Klimchuk 2006). The question is made even more difficult by the increasing evidence for an extremely fine-structured confined corona: the magnetic flux tubes that confine the bright coronal plasma are collections of a multitude of thin strands, of expected cross-section below the resolution of past and current coronal telescopes (Gomez et al. 1993; Vekstein 2009; Guarrasi et al. 2010). Finding direct signatures with current instruments is therefore not feasible for these inherently elusive mechanisms that occur on very small spatial scales. Indirect diagnostics are thus the only viable way at present. A feature that may strongly discriminate between gradual and impulsive heating release is the presence or absence of plasma at very high temperature. If we consider an active region, in order to keep the coronal loops at the typical temperature of 2-3 MK, we expect that occasional heat pulses should be so strong as to temporarily heat the plasma temperature to several MK, even above 10. Since we expect these heating episodes to be spread over all coronal structures, we might also search for a consequent minor but steady very hot plasma ( $\sim 6-10 \mathrm{MK})$ component. This might appear either as a hot tail or as a secondary peak to the hot side of the plasma emission measure distribution. Increasing evidence for this minor hot component has been collected in recent times, both with filter ratios or differential emission measure (DEM) reconstruction from imaging broadband instruments (XRT, e.g., Reale et al. 2009a, 2009b; McTiernan 2009; Schmelz et al. 2009) and with spectroscopic line analysis (single line or DEM reconstruction, e.g., Ko et al. 2009; Patsourakos \& Klimchuk 2009; Shestov et al. 2010; Sylwester et al. 2010), but it is still not conclusive for various reasons.

Spectroscopy would be ideal to reveal hot plasma but the moderate spatial and temporal coverage inhibits systematic campaigns and makes any detection or non-detection partial and limited (e.g., Warren et al. 2011). Also, DEM reconstructions typically suffer from severe limitations due to several factors such as the small number of line fluxes available in a narrow range of temperature, uncertainties in the element abundances, and, also in atomic data (e.g., Testa et al. 2011). This makes DEM reconstruction reliable on a large temperature scale at most, but much less on the details (e.g., Landi et al. 2012; P. Testa et al. 2012, in preparation). It then becomes appealing and reasonable to search for methods to discover hot plasma components with simple diagnostics and without addressing DEM reconstruction methods.

Recently, Reale et al. (2011) have used a different approach to infer the widespread presence of 6-8 MK plasma in the core of an active region. A model of multi-stranded pulse-heated coronal loop had predicted that if very hot component were present, it would be characterized by spatial distribution of its emission significantly sparser compared to the $2-3$ MK emission (Guarrasi et al. 2010). The Atmospheric Imaging Assembly (AIA; Lemen et al. 2012) on board the Solar Dynamics Observa- 
tory $(S D O)$ is based on normal-incidence optics and is equipped with six narrowband filters in the EUV band (94-335 $\AA$ ) that contain a few strong spectral lines that sample the solar corona in a wide temperature range, approximately between 0.5 and $10 \mathrm{MK}$. The instrument continuously monitors the full-disk corona with high cadence $(\lesssim 12$ s) and a pixel size of $\sim 0.6$ arcsec. One of the filters, the $94 \AA$ channel, includes a highly ionized Fe line, Fe XVIII, that is sensitive to plasma at temperatures in the range 6-8 MK. Reale et al. (2011) show that the bright $94 \AA$ emission in an active region core matches very well the filamented aspect predicted in the modeling paper. However, the $94 \AA$ passband nominally includes other spectral lines that make it sensitive also to plasma emitting in a range around $1 \mathrm{MK}$ (Boerner et al. 2012). The radically different morphology of the image obtained in the $171 \AA$ channel, containing a very strong Fe IX line sensitive to plasma at $\sim 1 \mathrm{MK}$, made the authors conclude that most of the plasma detected in the $94 \AA$ channel was in the hot branch. However, the lack of spectral resolution in the AIA observations leaves room from unknown line contributions that make the result not unequivocal.

Therefore, conclusive evidence of whether the $94 \AA$ bright emission in the core of active regions can be ascribed to hot plasma is expected to come from comparison with spectroscopic data. The Extreme-ultraviolet Imaging Spectrometer (EIS) on board Hinode (Culhane et al. 2007) is sensitive in the EUV band (in the 171-212 $\AA$ and 245-291 $\AA$ spectral ranges) and is able to provide images (spectroheliograms) in spectral lines thanks to rastering programs that take a few minutes to hours, depending on the target and field of view (FOV). In spite of the moderate spatial and temporal resolution, this instrument is appropriate for our task, since it has imaging capabilities, and its spectral range includes a Ca XVII line that is emitted by plasma at 6-8 MK (Patsourakos \& Klimchuk 2009; Ko et al. 2009; Warren et al. 2011). The scope of this work is to validate the detection of very hot plasma in active regions through the direct comparison of the morphology simultaneously observed with Hinode/EIS in the Ca XVII line and with SDO/AIA in the $94 \AA$ channel. Finding that well-defined bright structures have the same appearance in both observations would be very strong evidence that the bright $94 \AA$ emission in the AR cores really is due to plasma at 6-8 MK.

The task that we have tackled in this work is not so easy for various reasons: among the active regions that in the $94 \AA$ AIA channel show the presence of hot plasma, we have to find examples observed at the same time with EIS in the band including the Ca XVII line and including the hot plasma region in its FOV. Furthermore, the emission in the CaxVII line is not trivial to extract, because of blending with other lines, and consequently the deblended emission can be trusted only in regions where it is strong (Ko et al. 2009). Finally, the coalignment of the obtained EIS CaXVII image with the AIA images is also non-trivial.

In Section 2 we describe the data selection, co-alignment procedure, analysis methods, and results. We discuss our findings and draw our conclusions in Section 3.

\section{DATA SELECTION, ANALYSIS METHODS, AND RESULTS}

We searched for active region observations with simultaneous SDO/AIA imaging and Hinode/EIS spectral data, including the EIS short wavelength range (171-212 A), where the hot Ca XVII emission line lies $(\approx 192.85 \AA)$. We selected two active regions,
AR 11281 and AR 11289, observed close to disk center in 2011 September.

The selected EIS rasters are characterized by FOV of $120^{\prime \prime}$ $\times 160^{\prime \prime}$, slit width of $2^{\prime \prime}$, and exposure time of $60 \mathrm{~s}$ at each step (study acronym Atlas_60). The images are built up by stepping the $2^{\prime \prime}$ slit from solar west to east over a $\sim 1 \mathrm{hr}$ period. The study takes full spectra on both the EIS detectors from 171-212 A and 245-291 $\AA$. The EIS observations of AR 11281 have a start time of 2011 September 2 at 23:32UT, whereas the observations of AR 11289 started on 2011 September 13 at 10:37UT. The EIS data are processed with the eis_prep routine available in SolarSoft to remove the CCD dark current, cosmicray strikes on the $\mathrm{CCD}$, and take into account hot, warm, and dusty pixels. In addition, the radiometric calibration is applied to convert the data from photon events to physical units. The EIS routine eis_ccd_offset is also used to correct for the CCD offset. We selected simultaneous AIA data in the $171 \AA$, $335 \AA$, $94 \AA$, and $193 \AA$ passbands, and processed the level 1 data with the aia_prep routine, which performs image registration (coalignment and adjustments for the different plate scales and roll angle) and is also available as a part of SolarSoft.

In Figure 1 (left panels) we show the three-color full disk AIA images combining the $171 \AA, 335 \AA$, and $94 \AA$ intensities (green, blue, and red, respectively), at the start time of the EIS observations. The FOV of the EIS observations (120 arcsec $\times$ $160 \mathrm{arcsec})$ is marked on the full disk AIA images.

We co-aligned the data sets from the two instruments by applying a standard cross-correlation routine (tr_get_disp.pro which is part of the IDL SolarSoftware package) to the EIS images of the Fe XII $193 \AA$ A emission obtained from fitting the EIS spectra, and the $193 \AA$ AIA synthetic rasters (composite image, hereafter) built as follows: each vertical stripe is extracted from the AIA image closest in time to the time when the EIS slit was at that location. After co-aligning the time series AIA images in each channel (for the $1 \mathrm{hr}$ period corresponding to the EIS observations), and between channels, and to the EIS data, we created AIA composite images in each channel. In Figure 1 (middle panels) we show the three-color images, obtained by combining the $171 \AA, 335 \AA$, and 94 Åcomposite images in the EIS FOV. For each channel the intensity range chosen for the color scale covers a factor 40 , where the maximum is roughly half of the intensity maximum in the channel; the color scale is the same for the two data sets. Most features in the threecolor images appear dominated by a single color, the moss being a clear exception, as expected. For instance, the fan loops at the boundary of the AR are predominantly green (i.e., with dominant emission in the $171 \AA$ band), whereas blue (i.e., $335 \AA$ ) largely characterizes the emission of active region plasma. A few AR features are however dominantly pink-red, indicating significant emission in the $94 \AA$ and $335 \AA$ bands, with negligible contribution from the $171 \AA$ emission. The contribution of different spectral features to the AIA $94 \AA$ channel, as predicted using the CHIANTI database (Dere et al. 1997, 2009; the AIA responses as a function of temperature can be obtained using the SolarSoft routine aia_get_response), is dominated by Fe XVIII emission at the high temperature end $(\log T[K] \gtrsim 6.5)$, and by Fe $\mathrm{x}$ for warm plasma $(\log T[K] \sim 6)$. However, recent studies support the presence of significant additional contributions to the 94 A AIA passband missing from current atomic databases (e.g., Testa et al. 2012; also H. Warren 2012, private communication, has derived an empirical model of the warm contribution to the $94 \AA$ emission by scaling a combination of the $171 \AA$ and $193 \AA$ emission). 

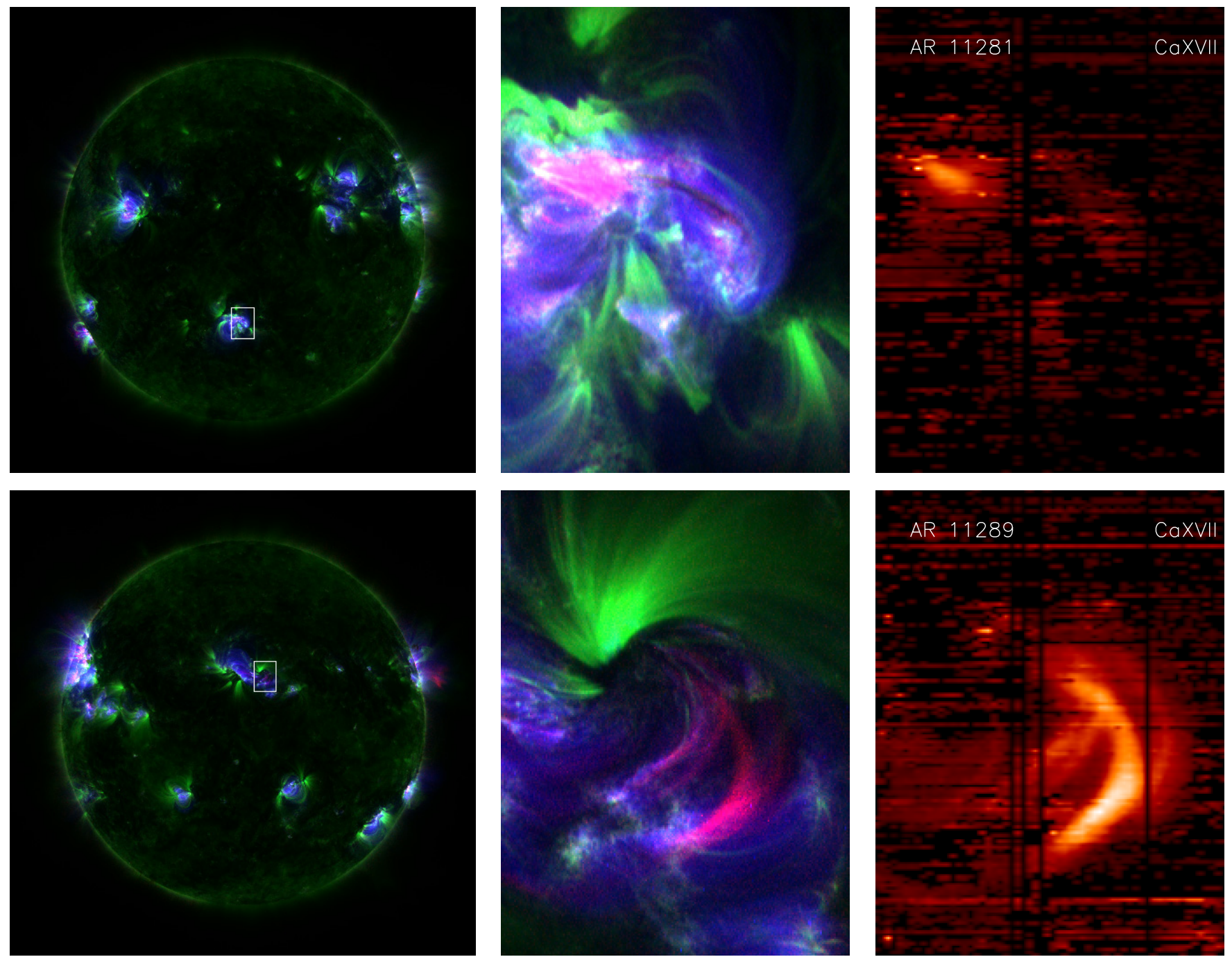

Figure 1. SDO/AIA and Hinode/EIS observations of AR 11281 (top row) and AR 11289 (bottom row). We show the AIA full disk data at the time of the beginning of the EIS observations, i.e., 2011 September 2 around 23:32UT and 2011 September 13 around 10:37UT for AR 11281 and AR 11289, respectively. Left: SDO/AIA three-color full disk image combining AIA observations in the $171 \AA$ (green), $335 \AA$ (blue), and $94 \AA$ (red) channels. The field of view of the EIS observations (120 arcsec $\times 160$ arcsec) is shown. Center: AIA three-color image for the EIS FOV (94 $\AA, 171 \AA$, and $335 \AA$ correspond to red, green, and blue, respectively, as in the left panels) created by building for each channel a composite image, where for each vertical slice in the EIS FOV we select the AIA data closest in time. Right: image of Ca XVII $192.85 \AA ̊$ emission from the EIS raster spectral data.

(A color version of this figure is available in the online journal.)

In order to validate the hypothesis that the $94 \AA$ emission for the "pink" features in the three-color image is dominated by Fe XVIII, i.e., emitted by hot plasma, we analyze the EIS spectra to measure the Ca XVII emission. We fit the Ca XVII lines using the procedure developed by Ko et al. (2009): we first fit the Fe XI lines at $\sim 188 \AA$, and then use their intensity to the model the Fe XI contribution to the $192 \AA$ feature which is a blend of Fe XI, O v, and Ca XVII. As discussed by Ko et al. (2009) the measurement of the Ca XVII emission from the multi-component fit to the $192 \AA$ a blend is deemed unreliable when its intensity is lower than $10 \%$ of the total intensity of the blend. Therefore, we assign zero value to the CaXVII intensity of those pixels meeting this condition. In the right panels of Figure 1 the maps of the Ca XVII intensity are shown, and, though somewhat noisy, they present clear evidence of confined bright features with morphology analogous to the pink features of the AIA color image.

Further more quantitative support is provided by scatter plots of the intensity in each AIA passband versus the Ca XVII intensity (Figure 2), indicating a clear correlation of the $94 \AA ̊$ intensity with the Ca XVII emission, which has no clear correlation instead with the two other AIA bands.

\section{DISCUSSION AND CONCLUSIONS}

The determination of the high temperature end $(\log T[K] \gtrsim$ 6.7) of the plasma thermal distribution is crucial to constraining the coronal heating (e.g., Klimchuk 2006; Reale 2010). In the literature, typical studies address this issue aiming at a full reconstruction of the emission measure distribution, based on either spectral or multi-filter (broadband/narrowband) imaging data (e.g., Del Zanna \& Mason 2003; Landi et al. 2009; Patsourakos \& Klimchuk 2009; Brooks et al. 2009; Warren \& Brooks 2009; Reale et al. 2009b; Sylwester et al. 2010; Testa et al. 2011). However, the determination of the DEM presents significant challenges because of difficulties inherent to the method (see, e.g., Craig \& Brown 1976; McIntosh 2000; Judge et al. 1997; Judge 2010; Landi \& Klimchuk 2010; Landi et al. 2012; P. Testa et al. 2012, in preparation), and limitations of available data, such as their spatial and temporal 

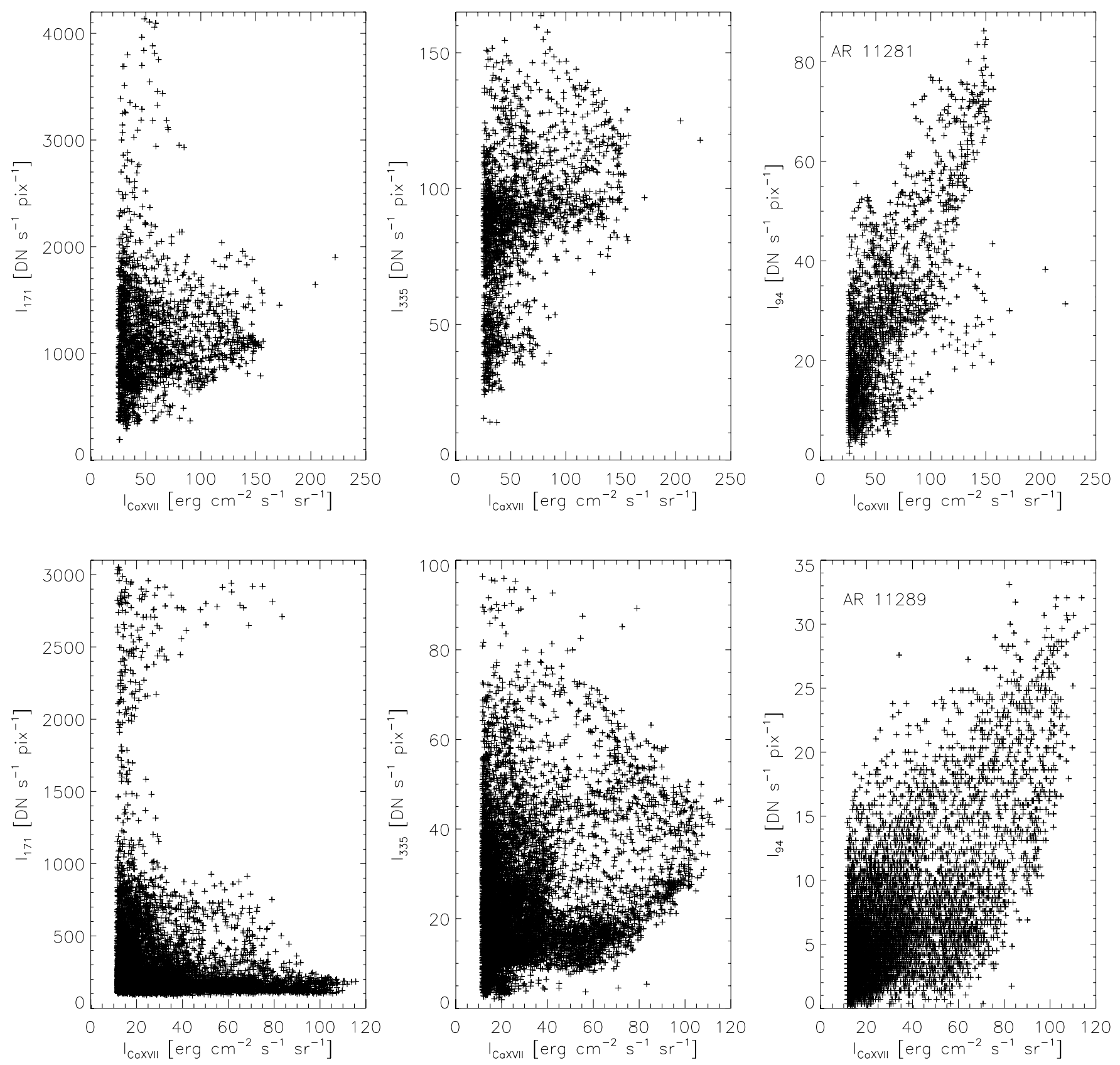

Figure 2. Intensity in the AIA $171 \AA$ (left), $335 \AA$ (middle), and $94 \AA$ (right) channels (in units of DN s ${ }^{-1}$ pixel $^{-1}$ ), plotted as a function of the intensity of the Ca XVII emission line (in units of erg cm $\mathrm{cm}^{-2} \mathrm{~s}^{-1} \mathrm{sr}^{-1}$ ). We selected only the pixels where the Ca XVII intensity was larger than $10 \%$ of the maximum value of Ca XVII emission in the entire EIS FOV.

coverage (especially for spectral observations), and temperature diagnostics (especially for imaging data).

In this Letter we adopted a different approach and investigated the diagnostic potential of $S D O /$ AIA imaging observations to diagnose the hot component $(\log T[K] \gtrsim 6.7)$ of the coronal plasma distribution. The comparison of AIA three-color images and EIS Ca XVII images shows a highly consistent scenario that confirms the presence of hot plasma in many AR cores, as pointed out in Reale et al. (2011). Figure 1 shows that the combination of the emission in the $171 \AA$, $335 \AA$, and $94 \AA$ AIA spectral bands is extremely effective in highlighting the hot plasma. We validate our findings by using EIS spectral data simultaneous to the selected AIA data, and establish the correspondence of the "hot" structures as singled out by the AIA three-color image with the structures with bright Ca XVII emission. We devised a color coding that clearly emphasizes the hot structures. Because of the full disk and high cadence nature of the AIA observations, one of the most notable implications of our findings is the capability of AIA to diagnose hot plasma at high spatial and temporal resolution, therefore determining the degree of its spatial variability and temporal evolution on timescales ranging from $12 \mathrm{~s}$, which is the typical cadence of standard AIA observations, to roughly two weeks, which is the crossing time from $\mathrm{E}$ to $\mathrm{W}$ limb.

We thank H. Warren for providing fitting routines for the EIS analysis, not included in the SolarSoft package. P.T. was supported by contract SP02H1701R from Lockheed-Martin, NASA contract NNM07AB07C to the Smithsonian Astrophysical Observatory, and NASA grants NNX10AF29G and 
3001762433. F.R. acknowledges support from Italian Ministero dell'Università e Ricerca and Agenzia Spaziale Italiana (ASI) contract ASI/INAFI/023/09/0 “Attività scientifica per l' analisi dati Sole e plasma - Fase E2/F." Hinode is a Japanese mission developed and launched by ISAS/JAXA, with NAOJ as domestic partner and NASA and STFC (UK) as international partners. It is operated by these agencies in co-operation with ESA and the NSC (Norway).

\section{REFERENCES}

Boerner, P., Edwards, C., Lemen, J., et al. 2012, Sol. Phys., 275, 41

Brooks, D. H., Warren, H. P., Williams, D. R., \& Watanabe, T. 2009, ApJ, 705, 1522

Craig, I. J. D., \& Brown, J. C. 1976, A\&A, 49, 239

Culhane, J. L., Harra, L. K., James, A. M., et al. 2007, Sol. Phys., 243, 19

Del Zanna, G., \& Mason, H. E. 2003, A\&A, 406, 1089

Dere, K. P., Landi, E., Mason, H. E., Monsignori Fossi, B. C., \& Young, P. R. 1997, A\&AS, 125, 149

Dere, K. P., Landi, E., Young, P. R., et al. 2009, A\&A, 498, 915

Gomez, D. O., Martens, P. C. H., \& Golub, L. 1993, ApJ, 405, 767

Guarrasi, M., Reale, F., \& Peres, G. 2010, ApJ, 719, 576
Judge, P. G. 2010, ApJ, 708, 1238

Judge, P. G., Hubeny, V., \& Brown, J. C. 1997, ApJ, 475, 275

Klimchuk, J. A. 2006, Sol. Phys., 234, 41

Ko, Y., Doschek, G. A., Warren, H. P., \& Young, P. R. 2009, ApJ, 697, 1956

Landi, E., \& Klimchuk, J. A. 2010, ApJ, 723, 320

Landi, E., Miralles, M. P., Curdt, W., \& Hara, H. 2009, ApJ, 695, 221

Landi, E., Reale, F., \& Testa, P. 2012, A\&A, 538, A111

Lemen, J. R., Title, A. M., Akin, D. J., et al. 2012, Sol. Phys., 275, 17

McIntosh, S. W. 2000, ApJ, 533, 1043

McTiernan, J. M. 2009, ApJ, 697, 94

Patsourakos, S., \& Klimchuk, J. A. 2009, ApJ, 696, 760

Reale, F. 2010, Living Rev. Sol. Phys., 7, 5

Reale, F., Guarrasi, M., Testa, P., et al. 2011, ApJ, 736, L16

Reale, F., McTiernan, J. M., \& Testa, P. 2009a, ApJ, 704, L58

Reale, F., Testa, P., Klimchuk, J. A., \& Parenti, S. 2009b, ApJ, 698, 756

Schmelz, J. T., Saar, S. H., DeLuca, E. E., et al. 2009, ApJ, 693, L131

Shestov, S. V., Kuzin, S. V., Urnov, A. M., Ul'Yanov, A. S., \& Bogachev, S. A 2010, Astron. Lett., 36, 44

Sylwester, B., Sylwester, J., \& Phillips, K. J. H. 2010, A\&A, 514, A82

Testa, P., Drake, J. J., \& Landi, E. 2012, ApJ, 745, 111

Testa, P., Reale, F., Landi, E., DeLuca, E. E., \& Kashyap, V. 2011, ApJ, 728, 30 Vekstein, G. 2009, A\&A, 499, L5

Warren, H. P., \& Brooks, D. H. 2009, ApJ, 700, 762

Warren, H. P., Brooks, D. H., \& Winebarger, A. R. 2011, ApJ, 734, 90 